\title{
Cerebrospinal fluid p-tau231 as an early indicator of emerging pathology in Alzheimer's disease
}

\author{
Nicholas J. Ashton, ${ }^{a, b, c, d, 1}$ Andréa L. Benedet, ${ }^{a, e, 1}$ Tharick A. Pascoal, ${ }^{e, f}$ Thomas K. Karikari, ${ }^{a, f}$ Juan Lantero-Rodriguez, ${ }^{a}$ \\ Wagner S. Brum, ${ }^{a, g}$ Sulantha Mathotaarachchi, ${ }^{e}$ Joseph Therriault, ${ }^{e}$ Melissa Savard, ${ }^{e}$ Mira Chamoun, ${ }^{e}$ Erik Stoops, ${ }^{,}$ \\ Cindy Francois, ${ }^{h}$ Eugeen Vanmechelen, ${ }^{h}$ Serge Gauthier, ${ }^{i, j}$ Eduardo R. Zimmer, ${ }^{g, k, l}$ Henrik Zetterberg, ${ }^{a, m, n, o}$ Kaj Blennow, $^{a, m}$ and \\ Pedro Rosa-Neto ${ }^{j, p_{*}}$
}

${ }^{a}$ Department of Psychiatry and Neurochemistry, Institute of Neuroscience and Physiology, the Sahlgrenska Academy at the University of Gothenburg, Mölndal, Sweden

${ }^{b}$ Wallenberg Centre for Molecular and Translational Medicine, University of Gothenburg, Gothenburg, Sweden

'King's College London, Institute of Psychiatry, Psychology and Neuroscience, Maurice Wohl Institute Clinical Neuroscience Institute, London, UK

${ }^{\mathrm{d}}$ NIHR Biomedical Research Centre for Mental Health and Biomedical Research Unit for Dementia at South London and

Maudsley NHS Foundation, London, UK

'Translational Neuroimaging Laboratory, McGill Centre for Studies in Aging, McGill University, Montreal, QC, Canada

fDepartment of Neurology and Psychiatry, University of Pittsburgh, Pittsburgh, USA

${ }^{g}$ Graduate Program in Biological Sciences: Biochemistry, Universidade Federal do Rio Grande do Sul (UFRGS), Porto Alegre,

Brazil

${ }^{\mathrm{h}}$ ADx NeuroSciences, Technologiepark 94, Ghent 9052, Belgium

'McGill University Research Centre for Studies in Aging, Douglas Research Institute, Le Centre intégré universitaire de santé et de services Sociaux (CIUSSS) de l'Ouest-de-l'Île-de-Montréal, Canada

jDepartment of Neurology and Neurosurgery, Director of the McGill University Research Centre for Studies in Aging, Psychiatry and Pharmacology and Therapeutics, McGill University, Montreal, QC, Canada

kDepartment of Pharmacology, Universidade Federal do Rio Grande do Sul (UFRGS), Porto Alegre, Brazil

'Graduate Program in Biological Sciences: Pharmacology and Therapeutics, Universidade Federal do Rio Grande do Sul

(UFRGS), Porto Alegre, Brazil

${ }^{m}$ Clinical Neurochemistry Laboratory, Sahlgrenska University Hospital, Mölndal, Sweden

${ }^{\mathrm{n}}$ Department of Neurodegenerative Disease, UCL Institute of Neurology, Queen Square, London, UK

${ }^{\circ}$ UK Dementia Research Institute at UCL, London, UK

${ }^{\mathrm{p}}$ Montreal Neurological Institute, Montreal, QC, Canada

\section{Summary}

Background Phosphorylated tau (p-tau) epitopes in cerebrospinal fluid (CSF) are accurate biomarkers for a pathological and clinical diagnosis of Alzheimer's disease (AD) and are seen to be increased in preclinical stage of the disease. However, it is unknown if these increases transpire earlier, prior to amyloid-beta (A $\beta$ ) positivity as determined by position emission tomography (PET), and if an ordinal sequence of p-tau epitopes occurs at this incipient phase

Methods We measured CSF concentrations of p-taui8I, p-tau2I7 and p-tau23I in I7I participants across the AD continuum who had undergone $A \beta\left(\left[{ }^{18} \mathrm{~F}\right] \mathrm{AZD} 4694\right)$ and tau $\left(\left[{ }^{\mathrm{I}} \mathrm{F}\right] \mathrm{MK} 6240\right)$ position emission tomography $(\mathrm{PET})$ and clinical assessment

Findings All CSF p-tau biomarkers were accurate predictors of cognitive impairment but CSF p-tau2I7 demonstrated the largest fold-changes in AD patients in comparison to non-AD dementias and cognitively unimpaired individuals. CSF p-tau23I and p-tau2I7 predicted $A \beta$ and tau to a similar degree but p-tau23I attained abnormal levels first. P-tau23I was sensitive to the earliest changes of $A \beta$ in the medial orbitofrontal, precuneus and posterior cingulate before global A $\beta$ PET positivity was reached

Interpretation We demonstrate that CSF p-tau23I increases early in development of AD pathology and is a principal candidate for detecting incipient $\mathrm{A} \beta$ pathology for therapeutic trial application

\footnotetext{
*Corresponding author at: Department of Neurology and Neurosurgery, Director of the McGill University Research Centre for Studies in Aging, Psychiatry and Pharmacology and Therapeutics, McGill University, Montreal, QC, Canada. E-mail address: pedro.rosa@mcgill.ca (P. Rosa-Neto).

${ }^{\mathrm{I}}$ These authors contributed equally to this work.
}

eBioMedicine 2022;76: 103836 Published online xxx https://doi.org/10.1016/j. ebiom.2022.103836 
Funding Canadian Institutes of Health Research (CIHR), Canadian Consortium of Neurodegeneration and Aging, Weston Brain Institute, Brain Canada Foundation, the Fonds de Recherche du Québec.

Copyright (c) 2022 Published by Elsevier B.V. This is an open access article under the CC BY-NC-ND license (http:// creativecommons.org/licenses/by-nc-nd/4.०/)

Keywords: Cerebrospinal fluid; Phosphorylated tau; Alzheimer's disease; Preclinical; Amyloid; Positron emission tomography

\section{Research in context}

\section{Evidence before this study}

In the context of dementia, elevated cerebrospinal fluid p-tau is highly indicative of Alzheimer's disease (AD). The residue phosphorylated at threonine 181 (ptau181) is often the validated biomarker used in routine biochemical assessments. However, recent evidence suggests other $\mathrm{p}$-tau epitopes to have greater diagnostic utility (p-tau217) or, importantly for disease prevention trials, an earlier elevation in the $A D$ continuum (p-tau231).

\section{Added value of this study}

Firstly, this is study confirms several aspects already partially described in the current literature; 1 ) all p-tau epitopes have high diagnostic capability for biomarker defined $A D, 2) p$-tau epitopes identify $A \beta$ and tau pathologies at all stages of the $A D$ continuum and 3) ptau217 exhibits larger fold-changes when symptomatic disease is apparent. Our novel finding suggests that CSF p-tau231 levels are raised earliest in preclinical AD. CSF $\mathrm{p}$-tau231 was shown to have markedly stronger focal associations $\mathrm{A} \beta$ PET retention in medial orbitofrontal, precuneus and posterior cingulate in $\mathrm{CU}$ individuals yet to reach the threshold of positive global $A \beta$ burden. Furthermore, we demonstrated that CSF p-tau231 displays the largest effect size in relation to $\mathrm{A} \beta$ deposition, both globally and regionally, and, amongst the biomarkers tested, is the first to achieve abnormal values.

\section{Implications of all the available evidence}

While the differences between p-tau epitopes are subtle, the early association of $\mathrm{p}$-tau231 with regional $\mathrm{A} \beta$ deposition is of fundamental importance for identifying individuals with subtle AD-related preclinical brain changes for therapeutic trials. It is anticipated that disease-modifying drug candidates have a better opportunity to show effectiveness if initiated before symptom onset or even before overt $A \beta$ plaque load. Thus, our evidence suggests that $\mathrm{p}$-tau231 has an advantage over other $\mathrm{p}$-tau epitope to highlight this early preclinical population.

\section{Introduction}

Neurofibrillary tangles (NFTs), primarily composed of abnormal hyperphosphorylated tau, are a key pathological hallmark of Alzheimer's disease (AD). ${ }^{\mathrm{I}}$ Increased concentrations of extracellular soluble phosphorylated tau (p-tau) and total tau (t-tau) constitute a reliable index of this intracellular process in mild cognitive impairment due to $\mathrm{AD}^{2,3}$ and $\mathrm{AD}$ dementia. ${ }^{2,4}$ NFTs are present many years after the deposition of extracellular amyloid-beta $(\mathrm{A} \beta)$ plaques but are more closely related to symptom onset and longitudinal studies have demonstrated that soluble p-tau and t-tau are increased already in preclinical disease (e.g., no cognitive impairment but evidence of AD pathology). ${ }^{5,6}$ Specifically, CSF concentrations of t-tau and p-tau are proposed to reflect neurodegeneration and tau pathology in the form of NFTs, respectively. ${ }^{7}$ However, these biomarkers remain at normal concentrations in other neurodegenerative disorders with substantial neurodegeneration, ${ }^{8-10}$ and also those with tau pathology. ${ }^{\mathrm{II}-\mathrm{I3}}$ Preclinical studies provide compelling evidence supporting early brain amyloidosis as a driver of tau hyperphosphorylation in AD. ${ }^{\mathrm{I}, \text { I5 }}$ Interestingly, evidence from AD neuronal culture models indicate that soluble $\mathrm{A} \beta$ oligomers $(\mathrm{A} \beta \mathrm{Os})$ induce tau hyperphosphorylation in multiple sites. ${ }^{\mathrm{I}, \mathrm{I7}}$ Indeed, the link between early cerebral $\mathrm{A} \beta$ deposition and CSF ptau elevations has been further supported by the fact that CSF tau species precede tau positron emission tomography (PET) abnormalities by a decade. ${ }^{\mathrm{I}, \mathrm{I9} 9}$ Thus, it is suggested that CSF p-tau biomarkers likely indicate an active process of tau secretion, and one that is correlated with cerebral $\mathrm{A} \beta$ deposition in early disease. ${ }^{20-22}$

CSF p-tau, in the context of AD, is often assumed to be the residue phosphorylated at threonine $\mathrm{I} 8 \mathrm{I}$ (ptaur8I). A multitude of mid-domain and C-terminal residues have also been described to be abnormally phosphorylated in the AD clinical spectrum ${ }^{\text {II,23-26 }}$ and studies specifically assessing their comparative diagnostic performance remain limited. Recent reports have suggested that CSF p-tau2I7 might better discriminate $\mathrm{AD}$ from other neurodegenerative diseases ${ }^{24,27-30}$ than CSF p-tauı8I. In addition, CSF p-tau2I7 correlates better with $\mathrm{A} \beta$ and tau PET imaging than CSF 
p-taui8r. ${ }^{24,30}$ These findings support an emerging framework suggesting that certain CSF tau phosphorylation species to predominate across the $\mathrm{AD}$ continuum. $^{24,27}$ We have recently reported that CSF ptau23I increases early in the AD continuum and, therefore, may detect preclinical $\mathrm{AD}$ among cognitively unimpaired (CU) individuals. ${ }^{3 \mathrm{I}}$ This is of fundamental importance for identifying individuals with subtle ADrelated preclinical brain changes for therapeutic trials, as it is anticipated that disease-modifying drug candidates have a better opportunity to show effectiveness if initiated before symptom onset or even before overt $\mathrm{A} \beta$ plaque load has been achieved. Indeed, a recent study in transgenic mice suggests that early removal of $\mathrm{A} \beta$ seeds, before $\mathrm{A} \beta$ deposition becomes detectable, led to a significant reduction of $\mathrm{A} \beta$ accumulation and downstream pathologies. $^{32}$ This emphasizes the need of a biomarker for detecting early $\mathrm{A} \beta$ seeds or the "pre-amyloid" phase. Therefore, it is imperative to investigate CSF p-tau epitopes associated with the earliest $\mathrm{A} \beta$ measures in $\mathrm{CU}$ to determine which of these early tau phosphorylation sites better reflects emerging $\mathrm{A} \beta$ pathology.

In order to address this knowledge gap, we investigated whether CSF p-tau epitopes (p-taui8r, p-tau2I7 and p-tau23I) are capable of identifying early $\mathrm{A} \beta$ pathology at the clinical, preclinical and pre-amyloid phases of AD. We also tested the performance of CSF p-taur8I, ptau2I7 and p-tau23I as biomarkers to predict clinical outcomes and $\mathrm{A} \beta$ and tau PET status. Based on our previous data, ${ }^{3 \mathrm{I}}$ we hypothesized that CSF p-tau23I associates with early $\mathrm{A} \beta$ pathology in brain regions that are affected early in the AD process. For these purposes, we performed voxel-wise analyses of the association between different CSF p-tau biomarkers and $\mathrm{A} \beta$ and tau PET in cognitively unimpaired (CU) and impaired (CI) individuals.

\section{Methods}

\section{Study design}

The main objective of this study was to investigate whether CSF p-tau epitopes can identify early $\mathrm{A} \beta$ deposition before $\mathrm{A} \beta$ PET positivity. We also aimed at comparing their performance to predict amyloid and tau pathologies indexed by PET in participants ranging within the AD continuum. This cross-sectional study was based on data from the Translational Biomarkers in Aging and Dementia (TRIAD) cohort, which is an observational and longitudinal biomarker-based study. TRIAD participants, mostly ranging in the $\mathrm{AD}$ spectrum, are followed yearly with detailed clinical and neuropsychological assessments, as well as with collection of fluid (blood, urine, saliva, and CSF) and acquisition of multiple imaging biomarkers. In addition to meeting standard diagnostic criteria, AD dementia participants had CDR between I and 2, subjects with mild cognitive impairment (MCI) had a CDR of 0.5 and CU subjects had a CDR of $\circ$. MCI participants without $\mathrm{A} \beta$ pathology are considered as non-AD neurodegenerative disease, together with participants clinically diagnosed with frontotemporal dementia (FTD; clinical diagnosis of behavioral or semantic variant of FTD, CDR score $>0.5$ and $\mathrm{A} \beta$ PET negative). This article includes I7I participants (27 young, <30 years; $82 \mathrm{CU}$; $20 \mathrm{MCI}$; 2I AD; 2I non-AD) from which CSF and PET imaging were available on October 20I9. For data description purposes, participants were sub-grouped according to their amyloid status as further described. For the imaging analysis, participants were initially evaluated according to their cognitive status as CU and CI.

\section{Ethics}

The TRIAD study was approved by The Research Ethics Board of the Montreal Neurological Institute as well as the Faculty of Medicine Research Ethics Office, McGill University, and all study participants provided written informed consent (Protocols: IUSMD I6-60 and I6-6I).

\section{CSF analysis}

CSF samples were collected by syringe and transferred to polypropylene tubes for centrifugation at $20{ }^{\circ} \mathrm{C}$, $2200 \mathrm{~g}$ for Io min. Samples were then distributed into ImL aliquots in polypropylene vials (Fisher Scientific Inc. Catalog \# 374I-WPiD-BR) and permanently stored at $-80{ }^{\circ} \mathrm{C}$ pending analyses.

All samples were analyzed for p-tauı8I, p-tau2I7 and p-tau23I. P-taui8I and p-tau2I7 were quantified by novel single molecule array (Simoa) assay that have been previously described. ${ }^{28,3 \mathrm{I}}$ In brief, rabbit polyclonal antibody specific for p-tau2I7 (\#44-744, Invitrogen) and mouse AT270 mouse monoclonal antibody (MNio50; Invitrogen, Waltham, MA, USA) were used as capture, conjugated to paramagnetic beads (\#I03207, Quanterix). The mouse monoclonal antibody Tauı2 (\#806502, BioLegend) raised against the N-terminal epitope 6-I8aa was used for detection. ${ }^{33}$ The assay calibrator was recombinant full-length tau-44I phosphorylated in vitro by glycogen synthase kinase $3 \beta$ (\#TO8$50 F N$, SignalChem). Calibrators and specimens were diluted with assay diluent (Tau 2.0 buffer; \#IOI556, Quanterix). Analytical validation and assay measurement protocol for both CSF p-tauI8I and CSF p-tau2I7 Simoa assays have been previously described. ${ }^{28}$ Both methods demonstrated intra and inter assay variation $<8 \%$. CSF p-tau23I was quantified using a research enzyme-linked immunosorbent assay (ELISA) assay using cis-conformational selective monoclonal antibody (ADx253, ADx NeuroSciences). Monoclonal mouse antibodies were generated using a I7-mer synthetic peptide, phosphorylated on hTau corresponding Thr23I, spanning the tau region $\mathrm{K}_{224} \operatorname{KVAVVR}_{(\mathrm{pT}) \mathrm{PPKSPSSAK}}{ }_{24} \mathrm{C}$ 
as a KLH-coupled antigen. Candidate hybridomas were selected on brain extracts of AD and control brain tissue. The final cloned and purified monoclonal antibody, ADx253, was characterized on synthetic peptides spanning the region T2I7 till S24I for its affinity, its phospho-specificity using both phosphorylated and nonphosphorylated peptides and its preferred selectivity in which proline at position 232 was replaced by a Pip (pipecolinic acid, piperidene-2-carboxylic acid, homoproline), to simulate cis-selectivity of ADx253. ${ }^{34} \mathrm{~A}$ pantau mouse mAb (ADx205, ADx NeuroSciences) with epitope in the mid tau region was used in biotinylated form as pairing antibody in the $\mathrm{p}$-tau23 I ELISA assay. ADx205 was recently fine mapped using overlapping linear synthetic peptides and the ADx205 antibody was found to bind between $\mathrm{R}_{\mathrm{I} 94}$ and $\mathrm{G}_{204}$ of tau44I or ${ }_{2} \mathrm{~N}_{4} \mathrm{R}$ tau sequence.

\section{Imaging analysis}

All participants have acquired $3 \mathrm{~T}$ TI-weighted images for co-registration purposes. In addition, a Siemens High Resolution Research Tomograph (HRRT) was used for PET imaging acquisitions, which occurred \pm 80 days from the CSF collection date (median $=53$ days). For $\mathrm{A} \beta \mathrm{PET}$, images were acquired 40-70 minutes post-injection of $\left[{ }^{18} \mathrm{~F}\right] \mathrm{AZD}_{4} 694$ and scans were reconstructed using the ordered subset expectation maximization (OSEM) algorithm on a 4-dimensional volume with 3 frames $(3 \times 600 \mathrm{~s}) .{ }^{35}$ For tau PET, $\left[{ }^{18} \mathrm{~F}\right]$ MK6240 scans were acquired 90-IIo minutes postinjection and the OSEM algorithm was also used for reconstruction on a $4 \mathrm{D}$ volume with 4 frames $(4 \times 300 \mathrm{~s}) \cdot{ }^{36}$ Additional pre-processing corrections were performed as described elsewhere. ${ }^{37}$ PET images were meninges and skull stripped, linearly and non-linearly registered to the ADNI template space and then spatially smoothed to achieve a final resolution of $8 \mathrm{~mm}$ FWHM. ${ }^{38}$ The inferior cerebellum and whole cerebellum gray matter were used as the reference regions for $\left[{ }^{18} \mathrm{~F}\right] \mathrm{MK} 6240$ and $\left[{ }^{18} \mathrm{~F}\right] \mathrm{AZD} 4694$, respectively.

Global A $\beta$ PET used averaged SUVR of the precuneus, cingulate, inferior parietal, medial prefrontal, lateral temporal, and orbitofrontal cortices ${ }^{39}$ and positivity was visually defined by two neurologists blinded to clinical diagnosis. An additional A $\beta$ PET SUVR cutoff value of I. 55 was estimated as previously described. ${ }^{4 \circ}$ A $\beta$ PET SUVR was also converted to Centiloid units ${ }^{4 \mathrm{I}}$ as previously described..$^{40,42}$ The PET SUVR cutoff value of I.55 corresponds to 24 Centiloids. Tau PET SUVR was globally estimated from a composite area including the transentorhinal (Braak stage I-II) and limbic (Braak IIIIV) cortices. ${ }^{43,44}$ Tau positivity was defined as 2.5 standard deviations (SD) higher than the mean global SUVR of the young participants ${ }^{38}$ (in this study $2.5 \mathrm{SD}=\mathrm{I} .03 \mathrm{SUVR}$ ).

\section{Statistical analysis}

All non-imaging statistical analyses were performed using the $\mathrm{R}$ programming language (version 3.4.3). Data distribution was visually inspected using histograms and those variables that did not follow a normal distribution were logio-transformed before parametric analysis when needed. Cross-sectional demographic and clinical data were assessed with linear models and $\chi^{2}$ tests. Spearman rank correlation tests were applied to evaluate the association between biomarkers and correlation coefficients were compared using the $\mathrm{R}$ package "Cocor". Linear regression models also tested the association between log-transformed CSF p-tau and other biomarkers always adjusting by age and gender. Similar models were also applied to evaluate group differences and, when necessary, Tukey honestly significant difference (HSD) test was used in the post hoc analysis. Receiver operating curves (ROC) provided both the area under the curve (AUC), for AD diagnosis or biomarker positivity, and the representative best value for accuracy at an optimal cutoff value. In addition to AUC, sensitivity and specificity, paired Delong's test ( $p$ ROC R package) was applied to statistically compare biomarker performance. Finally, imaging and CSF cutoffs were used to evaluate concordance between biomarkers.

Voxel-wise analyses were performed using VoxelStats. ${ }^{45}$ Voxel-based linear regression models evaluated the correlation between log transformed CSF values and PET biomarkers adjusting for age, sex, and diagnosis, when necessary. Random field theory ${ }^{46}$ was used to correct the resulting $t$ parametric maps for multiple comparisons (one-sided). To compare the effect of CSF biomarkers on the PET biomarkers, the adjusted Rsquared values of the models were averaged within ROIs encompassing only the voxels that were significantly associated to all CSF biomarkers simultaneously. The ROIs include precuneus, posterior cingulate, frontal orbital gyri, post central gyri and medial frontal gyri for $\mathrm{A} \beta$ PET, whilst for tau PET they were the average of Braak I-II, Braak III-IV and Braak V-VI staging regions.

CSF biomarkers were also plotted as a function of $\mathrm{A} \beta$ PET, which served as a proxy of disease progression. For that, $\mathrm{A} \beta$ PET values were given in Centiloid units and CSF biomarkers were first adjusted by age and sex and only then transformed in Z-score values based on the average and standard deviation of the $\mathrm{CU}$ population. Finally, a locally weighted polynomial regression method was employed, using the lowess function in R, with 0.6 smoother spam.

\section{Role of funding source}

This work was supported by the Weston Brain Institute, Canadian Institute of Health Research (CIHR), and Fonds de recherche du Québec - Santé. None of the funders had a role in the study design, data collection, data analyses, interpretation or writing of the report 


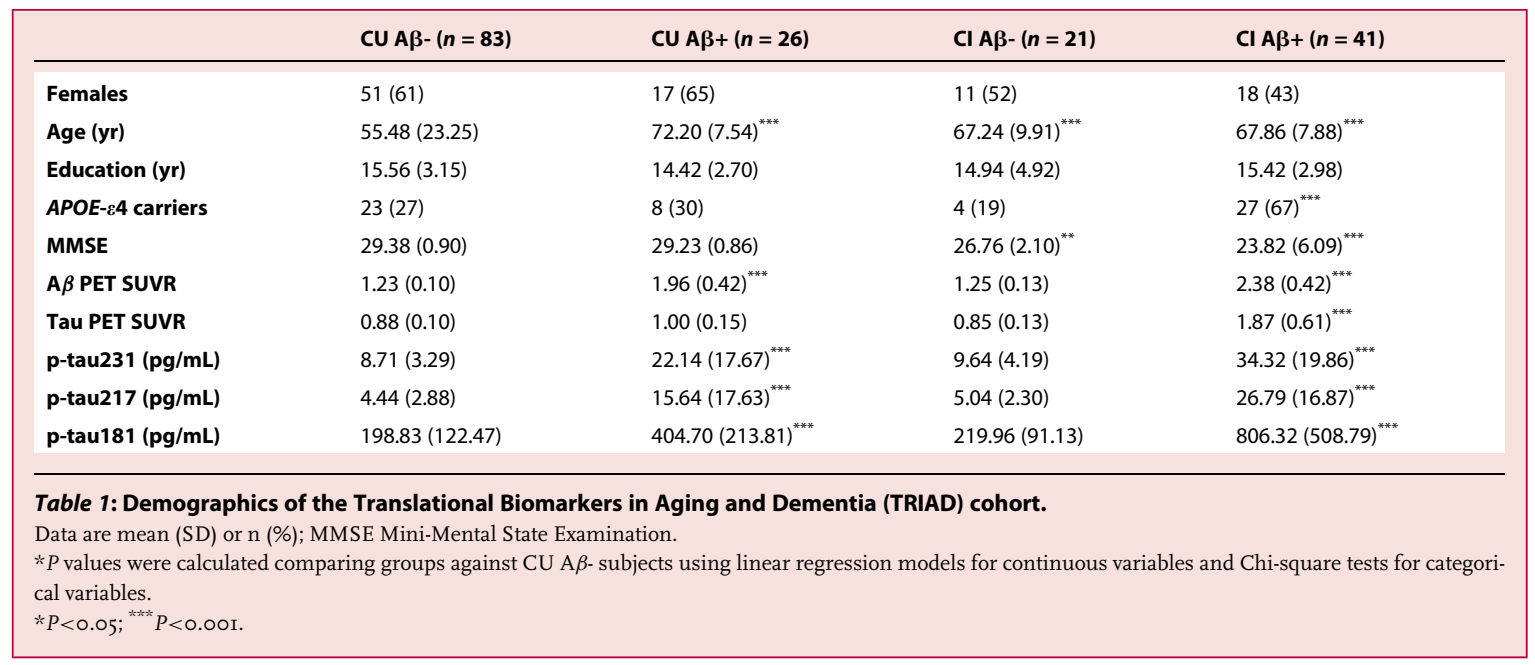

\section{Results}

\section{Participants}

This study included I7I participants, stratified as CU $(n=\operatorname{Iog}[23 \% \mathrm{~A} \beta \mathrm{PET}+])$ and CI $(n=62[66 \% \mathrm{~A} \beta \mathrm{PET}+])$ groups (Table I), with cross-sectional CSF (p-taui8I, ptau2I7, p-tau23I) and PET $\left(\left[{ }^{\mathrm{I}} \mathrm{F}\right] \mathrm{AZD} 4694\right.$ and $\left[{ }^{\mathrm{I}} \mathrm{F}\right]$ MK6240). The same participants were also categorized as $\mathrm{CU}(\mathrm{A} \beta+/-)$, MCI $(\mathrm{A} \beta+)$, $\mathrm{AD}$ and non-AD (supplementary table I). The mean age of the population was 62.77 years, with CI participants being older than $\mathrm{CU}(\mathrm{CU}=59.82$; $\mathrm{CI}=67.78 ; P<0.00 \mathrm{I}$ ) owing to a proportion of the $\mathrm{CU}$ being $<30$ years. As expected, the CI group had a lower MMSE scores, and higher $\mathrm{A} \beta$ PET and tau PET load as compared to the CU group (Table I). APOE- 84 carriers and males were also more common in the CI group. Older age was associated with higher levels of all CSF p-tau. There was no association between sex and p-tau biomarkers when adjusting for age and diagnosis.

\section{CSF p-tau biomarkers biomarker defined groups}

CSF p-tau biomarkers were highly correlated between each other, in the whole cohort, and in diagnostic categories groups (supplementary Figure ra-c) with the strongest association being between CSF p-tau2I7 and CSF p-tau23I in whole cohort $(r=0.92, P<0.001)$ and in the CI group $(r=0.93, P<0.00 \mathrm{I})$. Within the $\mathrm{CU}$ group, the strongest association was found between CSF p-taui8I and CSF p-tau23I $(r=0.86, P<0.00$ I [Spearman correlation]).

When assessing groups as either CU or CI, all CSF ptau biomarkers were significantly increased in $\mathrm{A} \beta+$ groups as compared to $\mathrm{A} \beta$ - groups. CSF p-tau23I (Figure ra) and CSF p-tau2I7 (Figure Ib) were significantly increased in CU A $\beta+$ as compared to CI $\mathrm{A} \beta$ - but this was not observed for CSF p-taui8I (Figure Ic). CSF p-tau2I7 was 6-fold higher in $\mathrm{CI} \mathrm{A} \beta+$ than in $\mathrm{CU} \mathrm{A} \beta$-, which was a significantly larger increase than other $\mathrm{p}$-tau biomarkers $\left(P_{p \text { - }}\right.$ tau $231_{1}=0.002 ; P_{p \text {-tau } 181}=0.0$ I; supplementary table 2$)$. Similarly, CSF p-tau2I7 demonstrated the largest fold increase between CI A $\beta+$ and CI A $\beta$ - (4.7-fold, $P<0.002$; supplementary table 2 ). However, this was not significantly different to other CSF p-tau biomarkers.

CSF p-tau biomarkers concentrations were also visualized by specific diagnostic categories (Figure $\mathrm{Id}-\mathrm{f}$ ) and fold change (supplementary table 2). This analysis further demonstrated that CSF p-tau2I7 had a superior fold change to other $\mathrm{p}$-tau biomarkers $(P<0.02)$ when specifically comparing MCI A $\beta+$ and $\mathrm{AD}$ dementia with CU A $\beta$ - (MCI A $\beta+$, 5.9-fold; AD, 7.I-fold) and non-AD neurodegenerative disorders (MCI A $\beta+, 4.8$-fold; $\mathrm{AD}$, 5.8-fold). CSF A $\beta 42 / 40$ groups differences are demonstrated in supplementary Figure 2.

Performance of CSF p-tau in clinically defined groups Next, we investigated the diagnostic accuracy of CSF ptau biomarkers in differentiating clinical categories which was predetermined by underlying $\mathrm{A} \beta$ and tau PET status (supplementary table 3). In a ROC analysis, CSF p-taui8I was the best performer in distinguishing between $\mathrm{CU}$ and $\mathrm{AD}$, when $\mathrm{A} \beta$ and tau PET status were unknown (AUC = 0.97; 95\% CI, 0.95-0.99). This significantly outperformed CSF p-tau23I $(P<0.0$ I [DeLong's]) but not CSF p-tau2I7 ( $P=0.13$ [DeLong's]). All biomarkers had high accuracies (AUC $>0.96$ ) in separating $\mathrm{AD}$ from non-AD and no biomarker was found to be statistically superior in this comparison. A noticeable decline in performance was observed for CSF p-taui8I when distinguishing $\mathrm{MCI}$ from non-AD (AUC $=0.83 ; 95 \% \mathrm{CI}, 0.67-0.99)$ and $\mathrm{MCI}$ from CU (AUC $=0.8 \mathrm{I} ; 95 \% \mathrm{CI}, 0.66-0.96)$ which was significantly different to p-tau23I and p-tau2I7 $(P<0.05$ 

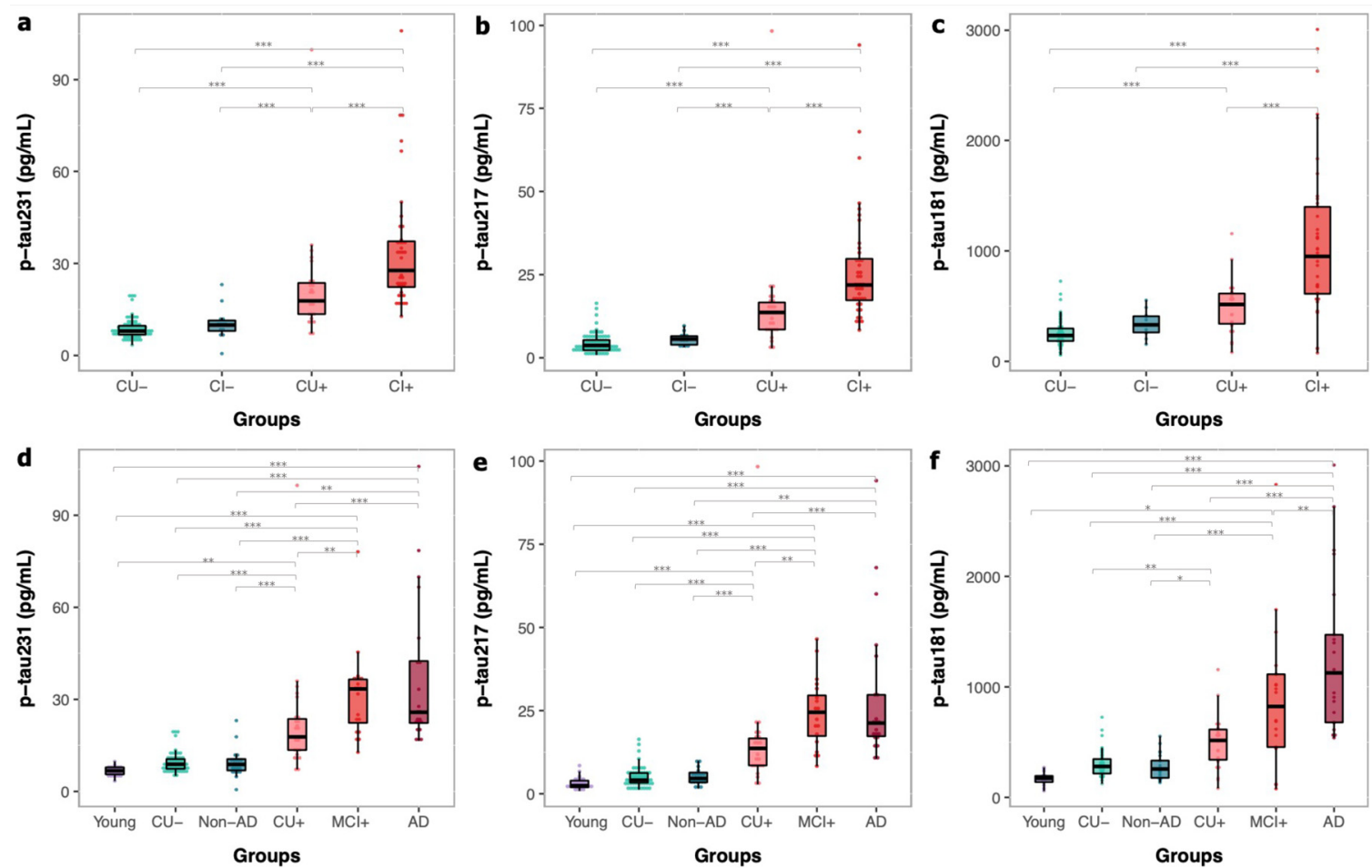

Figure 1. Phosphorylated tau CSF epitopes by group. Distribution of CSF biomarkers concentrations across groups showing higher biomarker levels associated with $A \beta$ positivity. $C U$-: cognitively unimpaired $A \beta$ negative; $C U+$ : cognitively unimpaired $A \beta$ positive; $\mathrm{Cl}-$ : cognitively impaired $\mathrm{A} \beta$ negative; $\mathrm{Cl}+$ : cognitively impaired $\mathrm{A} \beta$ positive; $\mathrm{MCl}+$ : mild cognitively impaired $\mathrm{A} \beta$ positive; $\mathrm{AD}$ : Alzheimer's disease dementia; Non-AD: mild cognitively impaired $A \beta$ negative and frontotemporal dementia (FTD). ${ }^{* * *} P<0.001$; ${ }^{* *} P<0.01 ;{ }^{*} P<0.05$ (Tukey HSD adjusted).

[DeLong's]). No such changes were observed for other biomarkers demonstrating that CSF p-tauI8I is not sensitive to early pathological changes but an accurate biomarker at late-stage disease.

\section{Associations between CSF p-tau biomarkers with tau PET}

Tau PET was performed in all I7I participants included in this study. High concentrations of all CSF p-tau biomarkers were associated with increased retention of $\left[{ }^{18} \mathrm{~F}\right] \mathrm{MK} 6240$ composite Braak stage I-IV. A similar correlation coefficient was observed between CSF p-tau23I $(r=0.74, P<0.001$, Figure 2a) and CSF p-tau2I7 $(r=0.73, P<0.00$ I, Figure $2 \mathrm{~b})$ with Braak stage I-IV. In contrast, significantly inferior correlations $\left(P_{\mathrm{p} \text {-tau23r vs }}\right.$ p-taui $\left.8 \mathrm{I}=0.004 ; P_{\mathrm{p} \text {-tau2I7 vs p-tauI8I }}=0.02\right)$ were observed for CSF p-tauisi $(r=0.66, P<0.00$ I, Figure $2 \mathrm{c})$. No significant differences were found between the CSF biomarkers when predicting tau PET positivity and all demonstrated comparable AUC values $(\mathrm{AUC}=0.86$ 0.93 , Figure $2 \mathrm{~d}$ ). This included CSF A $\beta 42 / 40$ which also had high accuracy to distinguish tau PET status (AUC 0.9I; 95\% CI, 86-97\%).

At the voxel level, CSF biomarkers were associated with higher $\left[{ }^{18} \mathrm{~F}\right] \mathrm{MK} 6240$ uptake in the inferior, medial and lateral temporal regions $\left(T_{\text {all }}>3 . \mathrm{I} 4 ; \quad P_{\text {all }}<0.05\right.$, Figure $2 \mathrm{E}$ ), with associated areas overlapping between CSF biomarkers. No marked difference was observed between CSF p-tau23I and CSF p-tau2I7, whilst CSF ptaui8I indicated weaker associations narrowed to reduced regions if compared to the other biomarkers (Figure 2e). When groups were evaluated as CI (Figure 2f) and CU (Figure 2g) separately, broad associations were found in the CI group $\left(T_{\mathrm{CI}}>3.25 ; P_{\mathrm{CI}}<0.05\right.$, Figure $2 \mathrm{f}$ ), where high concentrations of CSF biomarkers were associated with high $\left[{ }^{\mathrm{I} 8} \mathrm{~F}\right] \mathrm{MK} 6240$ binding in the temporal lobes, cingulate regions, and orbitofrontal cortices, encompassing Braak stage regions I-VI. A stronger relationship between $\left[{ }^{\mathrm{I} 8} \mathrm{~F}\right]$ MK6240 and CSF p-tau23I and CSF p-tau2I7 $\left(T_{\mathrm{CU}}>3 . \mathrm{I} 6 ; P_{\mathrm{CU}}<0.05\right)$ were found in CU participants, with significant associations confined to temporal regions corresponding to Braak stage regions I-IV (Figure 2g).

\section{Associations between CSF p-tau biomarkers with A $\beta$ PET}

$\mathrm{A} \beta$ PET was performed in all I7I participants included this study. In a similar findings to tau PET, correlation coefficients where strongest between $\left[{ }^{18} \mathrm{~F}\right] \mathrm{AZD} 4694$ 

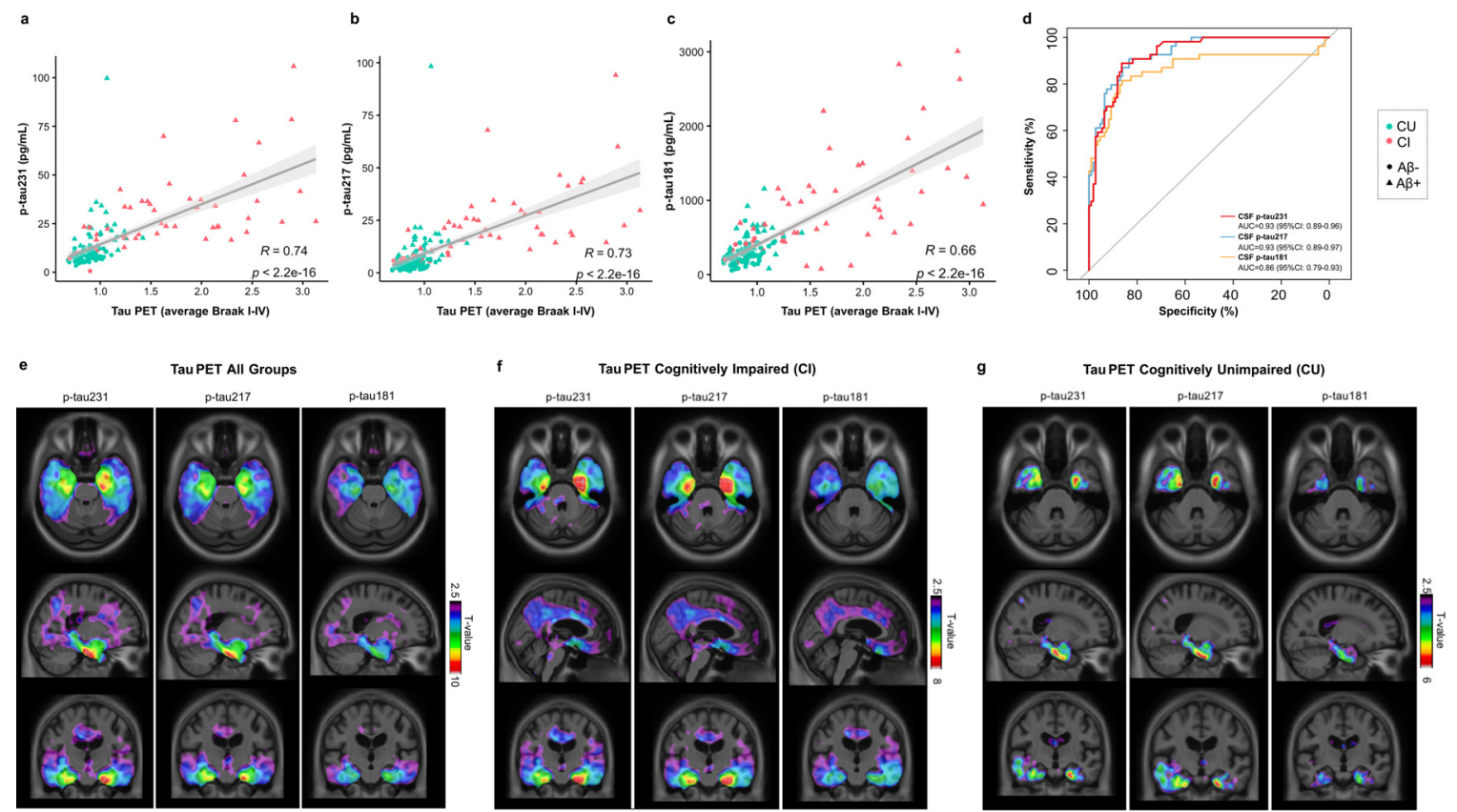

Figure 2. Phosphorylated tau CSF epitopes and Tau PET associations. Spearman rank correlations between CSF biomarkers and tau PET ( $\left.\left[{ }^{18} \mathrm{~F}\right] \mathrm{MK} 6240\right)$ across all groups show $\mathrm{p}$-tau231 as having the highest correlation coefficient, followed by $\mathrm{p}$-tau217 (a-c). The accuracy of CSF biomarkers in distinguishing tau PET status (positive vs negative) is evidenced by AUCs as shown in (d). T-parametric maps show the voxel-wise association between CSF biomarkers and $\left[{ }^{18} \mathrm{~F}\right]$ MK6240 in all participants (e) as well as within $\mathrm{Cl}(\mathrm{f})$ and $\mathrm{CU}$ (g). Models were adjusted for age and sex and RFT was used to account for multiple comparisons (significant t-values $>3.1$ ). CU: cognitively unimpaired; $\mathrm{Cl}$ : cognitively impaired.

global retention and CSF p-tau23I $(r=0.8 \mathrm{I}, P<0.00 \mathrm{I}$, Figure 3a) and CSF p-tau2I7 $(r=0.79, P<0.00 \mathrm{I}$, Figure $3 \mathrm{~b})$. Once more, correlations were inferior for CSF p-taui8I $(r=0.70, P<0.001$, Figure $3 \mathrm{c})$ which were significantly different to other $\mathrm{p}$-tau biomarkers $\left(P_{\text {p-tau23I vs p-taur8I }}<0.00 \mathrm{I} ; P_{\text {p-tau217 vs p-taur8I }}=0.0 \mathrm{II}\right)$. CSF P-tau23 ( $\mathrm{AUC}=0.95 ; 95 \% \mathrm{CI}, 0.92-0.98)$ and CSF ptau2I7 (AUC $=0.95 ; 95 \% \mathrm{CI}, 0.92-0.99)$ were significantly better predictors of $\mathrm{A} \beta$ PET status than CSF ptauI8I (AUC = 0.88; 95\% CI, 0.8I-0.94; both $P<0.0 \mathrm{I}$, Figure $3 \mathrm{~d}$ ). CSF A $\beta 42 / 40$ was also a significantly better predictor of $\mathrm{A} \beta$ PET status than p-tauI8I ( $\mathrm{AUC}=94 \%$; $95 \%$ CI: $\left.90-99 \% ; P_{A \beta_{42} / 40 \text { vs p-taur } 8 \mathrm{I}}=0.003\right)$. No significant difference was found between CSF p-tau231, CSF p-tau2I7 and CSF A $\beta 42 / 40$ in the prediction of $\mathrm{A} \beta$ PET status. To further investigate these associations, biomarker accuracy to detect $\mathrm{A} \beta$ positivity was evaluated within CU and CI groups separately (supplementary Figure 3). In CU individuals, CSF p-tau23I $(\mathrm{AUC}=0.9 \mathrm{I} ; 95 \% \mathrm{CI}, 0.84-0.98)$ was significantly superior to CSF p-tauI8I ( $\mathrm{AUC}=0.82 ; 95 \% \mathrm{CI}$, 0.7I$0.93 ; P=0.02$ ) but not CSF p-tau2I7 (AUC = 0.90; $95 \% \mathrm{CI}$, 0.83-0.98; $P=0.75$ ). Conversely, in CI individuals, no CSF p-tau was found to be significantly superior, although CSF p-tau2I7 had numerically the highest AUC (0.99; 95\% CI, 0.99-I.00). Performing a ratio of p-tau23I and p-tau2I7 with CSF A $\beta_{42}$ showed no statistically significance difference than p-tau alone. However, a numerical advantage was observed in $\mathrm{CU}$ participants (p-tau23I/A $\beta 42, \mathrm{AUC}=0.96 ; 95 \% \mathrm{CI}$, 9299\%; p-tau2I7/A $\beta 42, \mathrm{AUC}=95 \% ; 95 \% \mathrm{CI}, 9 \mathrm{I}-99 \%)$. A ratio of CSF A $\beta 42$ with p-tauI8I improved the prediction of amyloid status in CU participants (p-taur8I/ $\mathrm{A} \beta 42, \mathrm{AUC}=90 \% ; 95 \% \mathrm{CI}, 82-99 \% ; P_{p-\operatorname{tau} 1} 8_{1} / A \beta_{42}$ vs $\mathrm{p}$ taur $8 \mathrm{r}=0.03)$.

Results of the voxel-wise analysis showed high CSF biomarker levels being associated with high $\left[{ }^{\mathrm{I}} \mathrm{F}\right]$ AZD4694 binding in frontal, precuneus, posterior cingulate and temporal cortices $\left(T_{\text {all }}>3.14 ; P_{\text {all }}<0.05\right.$, Figure 3e). Even though there was a topographical overlap of the significantly associated regions between the CSF biomarkers evaluated, a markedly stronger association was observed for CSF p-tau23I when the whole sample was analyzed (Figure 3e). In the CI group analyses, there was no difference between the associations of $\left[{ }^{18} \mathrm{~F}\right] \mathrm{AZD} 4694$ with CSF p-tau23I and CSF p-tau-2I7 $\left(T_{\mathrm{CI}}>3.25 ; P_{\mathrm{Cl}}<0.05\right.$; Figure $\left.3 \mathrm{f}\right)$. In contrast, in the $\mathrm{CU}$ group, there was an evident difference between CSF ptau23I and the other biomarkers (Figure 3g), despite there not being an evident difference in the whole set of participants $\left(\mathrm{T}_{\mathrm{CU}}>3.16 ; \mathrm{P}_{\mathrm{CU}}<0.05\right)$. Voxel-wise analysis including $\mathrm{CSF} A \beta_{42} / 40$ as a comparator in $\mathrm{CI}$ and $\mathrm{CU}$ demonstrated in supplementary figure 4 and 5 . As an additional comparison between biomarkers in the $\mathrm{CU}$ 

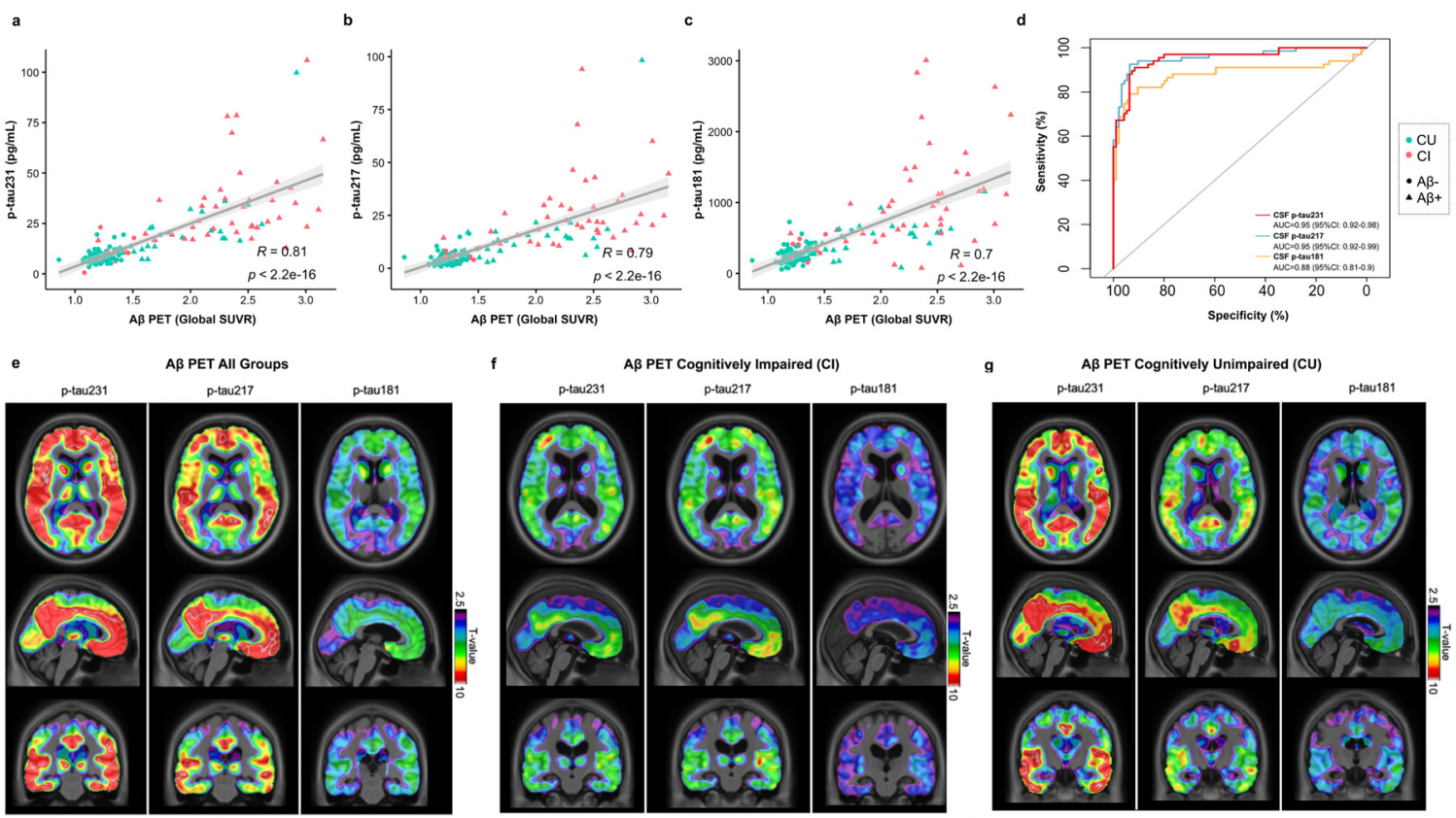

f

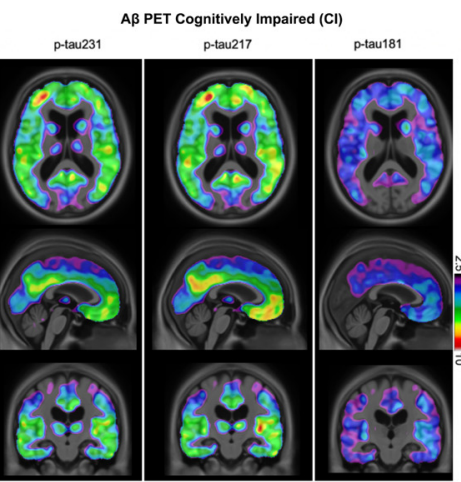

g

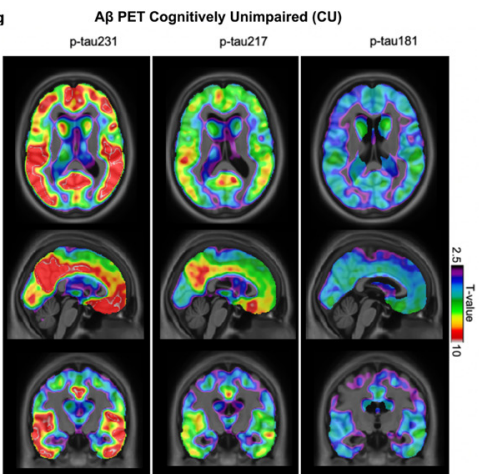

$\mathrm{h}$
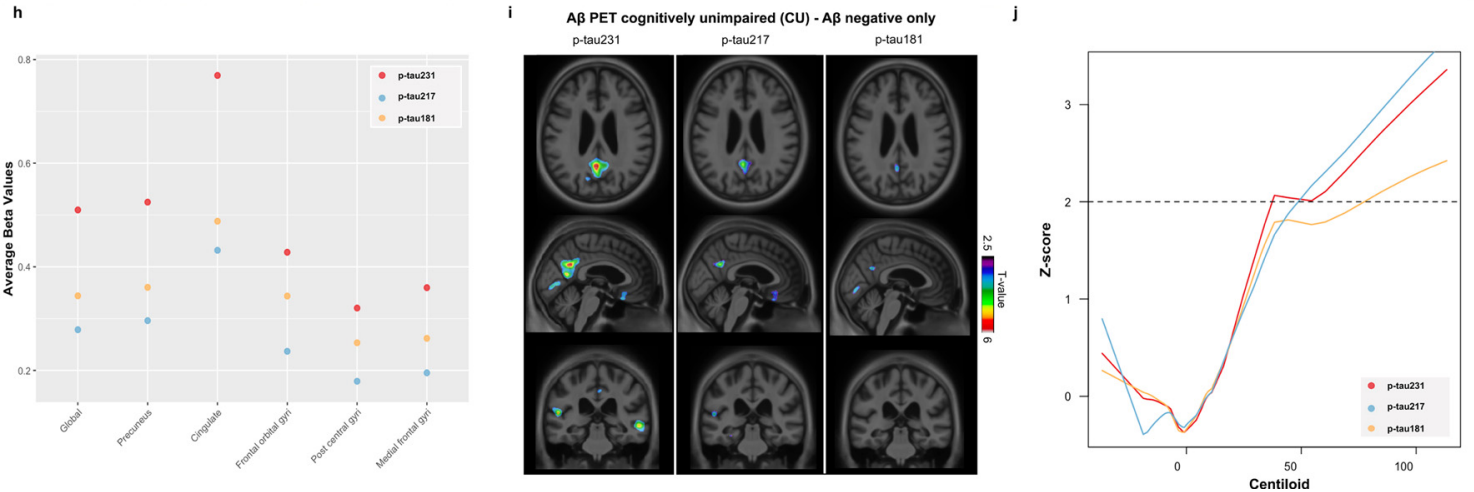

Figure 3. Phosphorylated tau CSF epitopes and A $\beta$ PET associations. Spearman rank correlations between CSF biomarkers and A $\beta$ PET ( $\left[{ }^{18}\right.$ F]AZD4694) across all groups show $p$-tau231 as having the highest correlation coefficient (a-c). The accuracy of CSF biomarkers in distinguishing $\mathrm{A} \beta$ PET status (positive vs negative) is evidenced by AUCs as shown in (d). T-parametric maps show the voxel-wise association between CSF biomarkers and $\left[{ }^{18} \mathrm{~F}\right]$ AZD4694 in all participants (e) as well as within $\mathrm{Cl}(\mathrm{f})$ and $\mathrm{CU}(\mathrm{g})$. Models were adjusted by age and sex and RFT was used to account for multiple comparisons (significant t-values $>3.1$ ). The dot plot (h) shows the average slope (beta) values by ROI for each of the CSF biomarkers. The beta value was derived from linear models that had $\mathrm{A} \beta$ PET as the outcome measure, the CSF biomarkers were the predictors and the covariates were sex and age. These models were performed at the voxel level and the CSF p-tau beta values of each voxel were averaged within ROls. Were included in the ROls only the voxels that had a significant association between $\mathrm{A} \beta$ PET and all of the CSF biomarkers evaluated here. T-parametric maps (i) show the voxel-wise association between CSF biomarkers ( $\mathrm{p}$-tau231, $\mathrm{p}$-tau217 and $\mathrm{p}$-tau181) and $\left[{ }^{18} \mathrm{~F}\right] \mathrm{AZD} 4694$ in A $\beta$-negative CU subjects. Models were adjusted for age and sex and RFT was used to account for multiple comparisons (significant $t$-values > 3.1). In addition, CSF p-tau biomarkers were plotted (j) as a function of A $\beta$ PET deposition (in Centiloids) in the CU group, which was used to infer AD pathology progression. Dashed line indicates the biomarker cut off for positivity. CU: cognitively unimpaired; Cl: cognitively impaired.

group, the average beta values from the linear models were computed at the voxel-level and then averaged within ROIs. For all the regions evaluated, CSF p-tau23I had the highest beta values as compared to the other biomarkers globally and in all ROIs (Figure $3 \mathrm{~h}$ ). To support these initial findings, we used $\mathrm{A} \beta$ PET (in Centiloids) as a proxy of $\mathrm{AD}$ progression and we estimated at which point the relationship between the CSF p-tau23I, CSF p-tau2I7 and CSF p-taur8I changes in relation to the disease in the whole study population (supplementary Figure 6). To perform this, we used locallyweighted polynomial regression analysis in which CSF biomarker levels were transformed into Z-scores and 2 Z-scores was defined as the cut-off value for biomarker 
positivity. No difference between the inflection point of these biomarkers was identified. Contrarily, CSF biomarkers showed to become abnormal at different pathological stages, with CSF p-tau23I status being positive at approximately Centiloid 37, followed by CSF p-taur8I at Centiloid 47 and CSF p-tau2I7 at Centiloid 50.

\section{CSF p-tau231 in emerging amyloid-beta pathology}

Considering the visually stronger association between CSF p-tau23I and $\left[{ }^{18} \mathrm{~F}\right] \mathrm{AZD}_{4} 694$ within the CU group (Figure 3g), as compared to the other CSF biomarkers, we further investigated how these associations would be within the subjects in the earliest possible process of $\mathrm{A} \beta$ accumulation. Therefore, we repeated the voxel-wise analysis only in individuals classified as $\mathrm{CU} A \beta$ (Figure 3i; supplementary Figure 7). CSF p-tau23I was the biomarker that best associated with $\left[{ }^{\mathrm{I} 8} \mathrm{~F}\right] \mathrm{AZD} 4694$ uptake $\left(T_{\mathrm{CU}-}>3.19 ; P_{\mathrm{CU}-}<0.05\right.$, Figure 3i) which was significantly superior to CSF p-tauzI7 and CSF p-tauI8I biomarkers in this analysis. These associated areas were very focal and included the medial orbitofrontal, precuneus and posterior/isthmus cingulate cortices. In order to further support the findings suggesting that CSF ptau23I best reflects the earliest $\mathrm{A} \beta$ dysmetabolism, we repeated locally-weighted polynomial regression analysis, and re-estimated at which point the relationship between the CSF p-tau23I, CSF p-tau2I7 and CSF ptaui8I changes in relation to the disease within the CU group (Figure 3i). Again, no significant difference was observed between the inflection point of these biomarkers however, abnormality of CSF p-tau23I were observed far earlier (Centiloid 38.3) than whilst CSF ptau2I7 and CSF p-taur8I (Centiloids $>50$ ). Thus, this analysis suggests CSF p-tau23I as the biomarker with the fastest preclinical increases which is the first to present abnormal levels as $\mathrm{A} \beta$ accumulates in the brain of emerging $\mathrm{AD}$ pathology.

\section{Discussion}

The present study examined the relationship between CSF p-tau biomarkers (p-tau23I, p-tau2I7 and p-taur8I) with amyloid and tau PET in I7I individuals across the AD continuum. We demonstrate that CSF p-tau23I and CSF p-tau2I7 biomarkers have a similar relationship with $\left[{ }^{18} \mathrm{~F}\right] \mathrm{MK} 6240$ and $\left[{ }^{18} \mathrm{~F}\right] \mathrm{AZD} 4694$, being both better predictors of PET status in comparison with CSF ptaui8I. At the voxel level, however, CSF p-tau23I had a markedly stronger relationship with $\mathrm{A} \beta$ pathology in $\mathrm{CU}$ individuals. Our main finding suggests that CSF ptau23I abnormality arises during the lag phase of $\mathrm{A} \beta$ protein aggregation in the brain as shown by markedly stronger focal associations of CSF p-tau23I and $\mathrm{A} \beta$ PET in $\mathrm{CU}$ individuals without positive global $\mathrm{A} \beta$ burden. Furthermore, we demonstrated that CSF p-tau23I displays the largest effect size in relation to $\mathrm{A} \beta$ deposition, both globally and regionally, and, amongst the biomarkers tested, is the first to achieve abnormal values.

The formation of NFT by the aggregation of tau is a fundamental hallmark of AD pathogenesis. However, in what way the differential release of soluble phosphorylated tau into the CSF relates to the development of $\mathrm{A} \beta$ and NFT pathologies, and consequently neurodegeneration, remains to be clarified. It is also unclear whether extracellular soluble phosphorylated tau is a driver of tau propagation in AD. ${ }^{47,48}$ Our data, like prior studies, ${ }^{18,28,31}$ shows an increase of CSF p-tau epitopes in the preclinical phase of the disease, prior to symptom onset and before substantial tau PET ligand retention. We also confirm that CSF p-tau do not increase in nonAD dementias or cognitive impairment with absent or minimal $\mathrm{A} \beta$ burden. Thus, in relation to sporadic $\mathrm{AD}$, this study adds further support to the hypothesis that CSF $\mathrm{p}$-tau is closely related to $\mathrm{A} \beta$-mediated tau release from neurons ${ }^{19}$ and challenges the notion of being simply a state marker of NFT. However, while it is presumed that p-tau secretion, and subsequent increase in CSF, occurs after globally aggregated $\mathrm{A} \beta$, our data shows that CSF p-tau already changes with subtle $\mathrm{A} \beta$ pathology and therefore occurs considerably earlier than previously anticipated. This is seemingly more apparent for CSF p-tau23I, which changes with $\mathrm{A} \beta$ deposition in the medial orbitofrontal, precuneus and posterior cingulate cortices before $\mathrm{A} \beta$ PET positivity is achieved. Thus, one could propose that increases in CSF p-tau23I are related to early $\mathrm{A} \beta$ seeds and could act as a key biomarker for the recently described "pre-amyloid phase" of $\mathrm{AD}$, which occurs before $\mathrm{A} \beta$ deposition threshold. ${ }^{22}$

CSF p-tau23I has been widely reported as a biomarker to detect $\mathrm{AD}$ at both the MCI and dementia stages of disease $\mathrm{e}^{\mathrm{II}, 49-53}$ but often it has been concluded these changes are not different from CSF p-taui8I when directly compared. ${ }^{54}$ Yet, most of these studies did not evaluate the cognitively unimpaired phases of the AD continuum. Previous neuropathological findings, which motivated the CSF assay development of ptau23I, highlighted that a key component of pre-tangle pathology was phosphorylation at threonine $23 \mathrm{I}^{55}$ and that CSF p-tau23I is an important discriminant of Braak o-I from more advanced Braak stages. ${ }^{56}$ Tau phosphorylation at threonine 23I is one of the earliest events in the cascade of phosphorylation, ${ }^{57}$ that modulates tubulin assembly. ${ }^{58}$ Interestingly, soluble $\mathrm{A} \beta \mathrm{O}$ s are known to enhance kinase activity triggering tau hyperphosphorylation at threonine 23I in primary neuronal cultures. ${ }^{\mathrm{I} 6}$ Therefore, now that we can monitor the in vivo development of $\mathrm{A} \beta$ and tau pathologies by PET, it is not surprising that our analysis concludes that CSF p-tau23I is an early marker of emerging AD pathology in the phase when only subtle brain amyloidosis is apparent. Although CSF p-tau23I demonstrated numerically identical AUC's to CSF p-tauzI7 in the prediction of $\mathrm{A} \beta$ PET 
at CU stage of the AD continuum, only CSF p-tau23I and not CSF p-tau2I7 was significantly superior to CSF p-tauı8I. Furthermore, at the voxel-wise level, CSF ptau23I demonstrated substantially higher associations with $\left[{ }^{18} \mathrm{~F}\right] \mathrm{AZD} 4694$ retention than CSF p-tau2I7 in CU individuals. We further performed a voxel-wise analysis in $\mathrm{CU}$ participants without prominent $\mathrm{A} \beta$ pathology $(\mathrm{A} \beta-$ ) and demonstrated focal associations of CSF ptau23I with emerging $\mathrm{A} \beta$ pathology in the medial orbitofrontal, precuneus and posterior/isthmus cingulate cortices which were stronger than what was observed for p-tau2I7 and absent for p-taur8I. Previously, Palmqvist et al. ${ }^{59}$ showed that $\mathrm{A} \beta$ fibrils begin to accumulate early in core regions of default mode network (DMN), namely precuneus, posterior cingulate cortex, and orbitofrontal cortex. This finding was even reported in individuals with seemingly normal levels of $\mathrm{A} \beta$ in both PET and CSF biomarkers (PET-/CSF-) but who later progressed to exhibiting abnormal levels of CSF $\mathrm{A} \beta$ (PET-/CSF+). This demonstrates that $\mathrm{A} \beta$ fibrils start to accumulate predominantly within certain parts of the $\mathrm{DMN}$ in preclinical AD and our data reveals that CSF ptau23I is a stronger correlate to these early accumulating regions. Consequently, CSF p-tau23 I could be a useful indicator of early $\mathrm{A} \beta$ deposition, which can have practical implications for highlighting early AD risk and enrich the enrollment of individuals in the pre-amyloid phase in clinical trials. This was further substantiated by CSF p-tau23I having the largest beta values in relation to $\mathrm{A} \beta$ deposition and seemingly becoming abnormal before CSF p-tau2I7 and CSF p-taui8I when the pathophysiological progression was delineated by $\mathrm{A} \beta$ PET Centiloids. P-tau23I levels in blood have also recently been demonstrated to increase earlier than $\mathrm{p}$ tauı $8{ }^{60}$ (Tissot et al., 2022), however, a direct comparison with p-tauzI7 in early preclinical disease is not currently available.

$\left[{ }^{\mathrm{I}} \mathrm{F}\right] \mathrm{MK} 6240$ detects paired helical filament (PHF) tau and has shown a high binding affinity for brain homogenate rich in NFTs. ${ }^{61}$ CSF p-tau, regardless of phosphorylation site, has been considered as a biomarker of NFT pathology, while other studies propose CSF p-tau as a state marker for brain tau phosphorylation, ${ }^{62}$ yet the direct association between CSF p-tau and tau PET has been inconsistent. Increases in CSF p-tau occur before both tau PET ${ }^{20,63,64}$ and established measures of neuronal death (e.g., neurofilament light, magnetic resonance imaging or $\left[{ }^{18} \mathrm{~F}\right] \mathrm{FDG}$ PET), thus CSF ptau cannot merely reflect NFT leakage from dying neurons. Instead, evidence now documents active secretion of tau in normal and disease conditions. ${ }^{19,65}$ In this study, we found that CSF p-tau23I and CSF p-tau2I7 associated with $\left[{ }^{18} \mathrm{~F}\right] \mathrm{MK} 6240$ to the same degree in the whole group and at both $\mathrm{CI}$ and $\mathrm{CU}$ stages, whereas CSF p-taui8I had weaker associations. In agreement to this, Janelidze et al. previously described that CSF ptau2I7 had a consistently greater regional association to tau PET than CSF p-taui8I but CSF p-tau23I was not analyzed in this particular study. Our results are also in line with data suggesting that p-tau2I7 is a superior clinical biomarker. While no statistical superiority was demonstrated for p-tau2I7 over p-tau23I, we did observe higher fold changes between AD and all other clinical groups for $\mathrm{p}$-tau2I7 which is consistent with more recent findings. ${ }^{30}$

On the basis of the evidence presented above, we propose a theoretical framework in which CSF p-tau epitopes become abnormal in a temporally ordered manner as the disease progresses (Figure 4). Specifically, CSF ptau23 I becomes abnormal first and seems the principal p-tau candidate to identify early $\mathrm{A} \beta$ seeds in the recently proposed "pre-amyloid phase", ${ }^{2}$ which is currently seen as the optimal period for therapeutic intervention. As $\mathrm{A} \beta$ accumulates toward $\mathrm{A} \beta$ positivity, other $\mathrm{p}$-tau epitopes (p-tau2I7 and p-taui8I) then become abnormal with p-tau2I7 changing at quicker rate. At the time of tau positivity, during neurodegeneration and cognitive abnormalities phases, CSF p-tau epitopes have largely reached a plateau. ${ }^{66}$

\section{Limitations}

We are aware of limitations to this study. Firstly, to definitively describe an ordinal sequence of CSF p-tau biomarkers, from pre-amyloid to symptomatic phases of the disease, longitudinal studies are required. Further to this, our main finding of early of pre-amyloid changes of p-tau23I is based on $83 \mathrm{CU} \mathrm{A} \beta$ - individuals and should be replicated in an independent cohort which is not enriched for AD with exclusion criteria's. Using the ratio of $\mathrm{A} \beta 42$ to $\mathrm{p}$-tau did not largely change our results but produced higher AUC's, which should be further investigated in larger cohort sizes or utilized if the optimal p-tau is not available, as highlighted in this study. Furthermore, the use of cross-sectional A $\beta$ PET Centiloids as a proxy of time in the disease was employed and while a gradual $\mathrm{A} \beta$ accumulation is observed though $\mathrm{AD}$ development, it is not certain that a greater $\mathrm{A} \beta$ PET SUVR is indicative of more advanced disease state. CSF p-tau biomarkers in this study are compared on two different platforms, with differing detection and capture antibody configurations: N-terminally derived CSF ptaui8I and CSF p-tau2I7 on the Simoa platform, Midterminal CSF p-tau23I by ELISA. Thus, it cannot be ruled out that subtle changes in biomarkers performances are determined by platform differences or antibody superiority. Yet, we believe this is unlikely given that our most promising finding is derived from a traditionally less sensitive technique. In addition, the ADx253 antibody utilized in the p-tau23I ELISA was determined to simulate cis-selectivity however it cannot be determined the specific abundance of the toxic cis p-tau23I species in this assay format. 


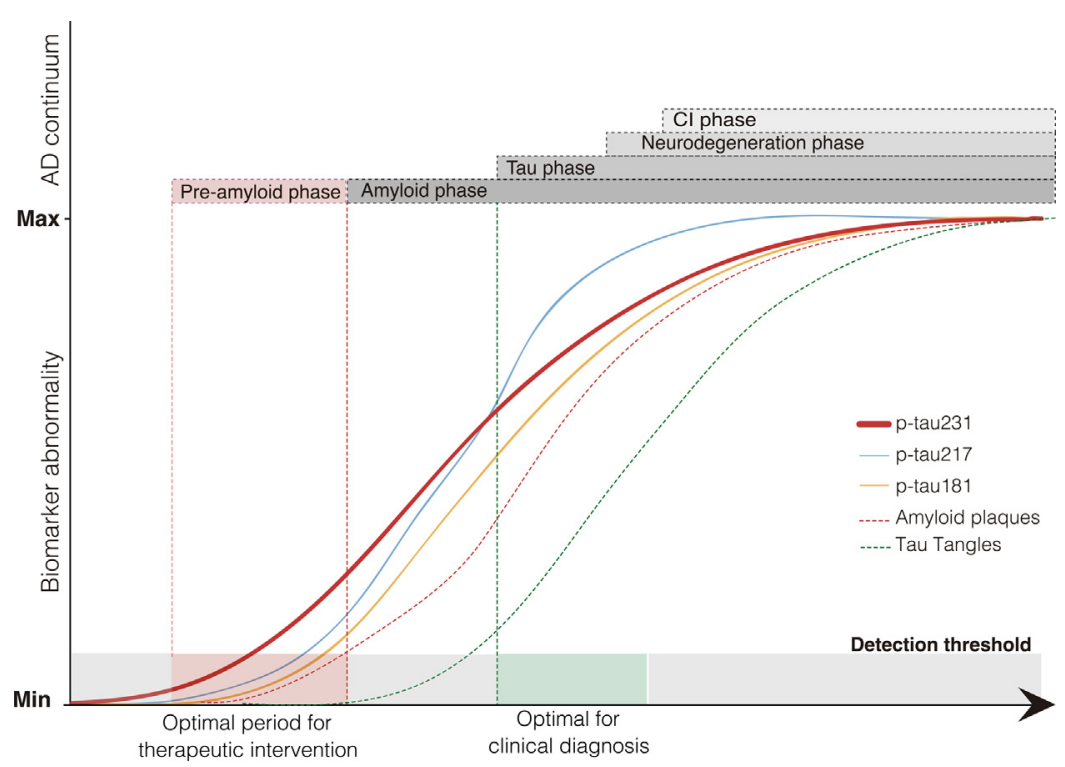

Figure 4. P-tau231 as an early biomarker of $A \beta$ pathology. The theoretical framework proposed presents a temporarily ordered progression of CSF p-tau epitopes in the context of the AD continuum, in which we hypothesize CSF p-tau231 as the first biomarker to become altered in the pre-amyloid phase, being then followed by p-tau217 and p-tau181 at pre-clinical and clinical stages.

In conclusion, this study documents the pronounced preclinical increases of CSF p-tau biomarkers but specifically highlights CSF p-tau23I which begins to associate with $\mathrm{A} \beta$ deposition before the threshold of $\mathrm{A} \beta$ PET positivity has occurred and strongly associates with known early $\mathrm{A} \beta$ accumulating regions in the DMN. This finding further supports a model of active soluble tau release by cells into the CSF which is related to early $\mathrm{A} \beta$ deposition, likely induced by early $\mathrm{A} \beta$ seeds. Thus, CSF p-tau23I is a novel candidate for detecting early and emerging $\mathrm{A} \beta$ pathology in individuals for the recruitment into therapeutic trials, act as a target engagement biomarker to monitor the effect of $\mathrm{A} \beta$ therapeutics or supports the notion of therapeutically targeting tau epitopes in preclinical disease before tau aggregation can be visualized by tau PET.

\section{Contributors}

NJA and ALB are equal ist co-authors. KB and PR-N are equal senior co-authors.

NJA, ALB, TAP, TKK, HK, OH, KB and PR-N conceived the study. Data acquisition was achieved NJA, TKK, JLR. NJA, ALB and WSB performed statistical analysis. KB and PR-N verified the underlying data. ALB, TAP, SJ, JT, MS, MC PR-N designed and implemented MRI and PET acquisition protocols, as well as performed image processing and quality control. SG, HZ, KB and PR-N recruited participants, and collected clinical data. NJA, ALB, TAP, TKK, ERZ, SG, HZ, KB and PR-N interpreted the data. NJA, ALB, ERZ, KB and PR-N drafted the initial manuscript. All authors contributed to revision and editing of the manuscript.

\section{Declaration of interests}

EVM is the Chief scientific officer in ADx Neurosciences. ES and CF are employees of ADx NeuroSciences. SG has served as a consultant for TauRx, Biogen Canada Member and Roche Canada. HZ has served at scientific advisory boards for Denali, Roche Diagnostics, Wave, Samumed, Siemens Healthineers, Pinteon Therapeutics and $\operatorname{CogRx}$, and has given lectures in symposia sponsored by Fujirebio, Alzecure and Biogen. Alzheimer's Association Global Biomarker Standardization Consortium chair and cofounder of Brain Biomarker Solutions in gothenburg AB (BBS) which is a part of the $\mathrm{GU}$ ventures Incubator program. KB has served as a consultant, at advisory boards, or at data monitoring committees for Abcam, Axon, Biogen, JOMDD/Shimadzu. Julius Clinical, Lilly, MagQu, Novartis, Roche Diagnostics, and Siemens Healthineers, and is a co-founder of Brain Biomarker Solutions in Gothenburg $A B$ (BBS), which is a part of the GU Ventures Incubator Program. CF, and ES and are employee of ADx NeuroSciences, Gent, Belgium, EVM is a co-founder of $\mathrm{ADx}$ NeuroSciences. The other authors declare no competing interest.

\section{Acknowledgements}

The authors thank all participants of the study and staff at University of Gothenburg, Sahlgrenska University Hospital, McGill University Research Centre for Studies, and Montreal Neurological Institute who supported this project. We thank Cerveau Technologies for MK6240 , and to GE Healthcare for providing the precursor of flutemetamol, and Roche for providing the precursor 
of RO948. We also thank Kimberley Mauroo for her assistance for the ADx205 epitope mapping. NJA was supported by the Wallenberg Centre for Molecular and Translational Medicine, Swedish Alzheimer Foundation, Hjärnfonden, the Swedish Dementia Foundation (Demensfonden) and Gamla Tjänarinnor. TAP was supported by the Alzheimer Society Research Program and the Canadian Consortium on Neurodegeneration in Aging. TKK is supported by the BrightFocus Foundation (\#A20208I2F), the Swedish Alzheimer Foundation (Alzheimerfonden; \#AF-930627), the Swedish Brain Foundation (Hjärnfonden; \#FO2O20-0240), the Swedish Parkinson Foundation (Parkinsonfonden; \#I252/ 20), the Swedish Dementia Foundation (Demensförbundet), Gamla Tjänarinnor Foundation, the Aina (Ann) Wallströms and Mary-Ann Sjöbloms Foundation, the Agneta Prytz-Folkes \& Gösta Folkes Foundation (\#2020-OOI24), the Gun and Bertil Stohnes Foundation, and the Anna Lisa and Brother Björnsson's Foundation. HZ is a Wallenberg Scholar supported by grants from the Swedish Research Council (\#2018-02532), the European Research Council (\#68I7I2), Swedish State Support for Clinical Research (\#ALFGBG-72093I), the Alzheimer Drug Discovery Foundation (ADDF), USA (\#201809-2016862), the European Union's Horizon 2020 research and innovation programme under the Marie Skłodowska-Curie grant agreement No 860I97 (MIRIADE), and the UK Dementia Research Institute at UCL. KB was supported by the Alzheimer Drug Discovery Foundation (ADDF; \#RDAPB-20I809-20I66I5), the Swedish Research Council (\#20I7-009I5), the Swedish Alzheimer Foundation (\#AF-74288I), Hjärnfonden, Sweden (\#FO2OI7-0243), and a grant (\#ALFGBG-715986) from the Swedish state under the agreement between the Swedish government and the County Councils, the ALF-agreement. TRIAD is supported by the Canadian Institutes of Health Research (CIHR) [MOP-II-5I-3I; RFN I52985, I598I5, I62303], Canadian Consortium of Neurodegeneration and Aging (CCNA; MOP-II-5I-3I -team I), Weston Brain Institute, Brain Canada Foundation (CFI Project 34874; 33397), the Fonds de Recherche du Québec - Santé (FRQS; Chercheur Boursier, 2020-VICO-2793I4). TAP, P.R-N and SG are members of the CIHR-CCNA Canadian Consortium of Neurodegeneration in Aging.

\section{Data sharing statement}

All data presented in this study is available upon request to the corresponding author. Data is not publicly available as it contains information that could compromise the privacy of research participants.

\section{Supplementary materials}

Supplementary material associated with this article can be found in the online version at doi:Io.IOI $6 / j$. ebiom.2022.103836.

\section{References}

I Scheltens P, Blennow K, Breteler MM, et al. Alzheimer's disease. Lancet. 2016;388(10043):505-517.

2 Olsson B, Lautner R, Andreasson U, et al. CSF and blood biomarkers for the diagnosis of Alzheimer's disease: a systematic review and meta-analysis. Lancet Neurol. 2016;15(7):673-684.

3 Hansson O, Zetterberg H, Buchhave P, Londos E, Blennow K, Minthon L. Association between CSF biomarkers and incipient Alzheimer's disease in patients with mild cognitive impairment: a follow-up study. Lancet Neurol. 2006;5(3):228-234.

4 Shaw LM, Vanderstichele H, Knapik-Czajka M, et al. Cerebrospinal fluid biomarker signature in Alzheimer's disease neuroimaging initiative subjects. Ann Neurol. 2009;65(4):403-4I3

5 Vos SJ, Xiong C, Visser PJ, et al. Preclinical Alzheimer's disease and its outcome: a longitudinal cohort study. Lancet Neurol. 2013;I2 (I0):957-965

6 Schindler SE, Li Y, Todd KW, et al. Emerging cerebrospinal fluid biomarkers in autosomal dominant Alzheimer's disease. Alzheimers Dement. 2019;I5(5):655-665.

7 Jack Jr. CR, Bennett DA, Blennow K, et al. NIA-AA Research Framework: Toward a biological definition of Alzheimer's disease. Alzheimers Dement. 20I8;14(4):535-562.

8 Riemenschneider M, Wagenpfeil S, Vanderstichele H, et al. Phospho-tau/total tau ratio in cerebrospinal fluid discriminates Creutzfeldt-Jakob disease from other dementias. Mol Psychiatry. 2003;8 (3):343-347.

9 Skillback T, Rosen C, Asztely F, Mattsson N, Blennow K, Zetterberg H. Diagnostic performance of cerebrospinal fluid total tau and phosphorylated tau in Creutzfeldt-Jakob disease: results from the Swedish mortality registry. JAMA Neurol. 20I4;7I(4):476-483.

Io Schoonenboom NS, Reesink FE, Verwey NA, et al. Cerebrospinal fluid markers for differential dementia diagnosis in a large memory clinic cohort. Neurology. 2012;78(I):47-54.

II Hampel H, Buerger K, Zinkowski R, et al. Measurement of phosphorylated tau epitopes in the differential diagnosis of Alzheimer disease: a comparative cerebrospinal fluid study. Arch Gen Psychiatry. 2004; 6I(I):95-IO2.

I2 Leuzy A, Smith R, Ossenkoppele R, et al. Diagnostic performance of RO948 F I8 tau positron emission tomography in the differentiation of Alzheimer disease from other neurodegenerative disorders. JAMA Neurol. 2020.

I3 La Joie R, Bejanin A, Fagan AM, et al. Associations between [(I8)F] AVI45I tau PET and CSF measures of tau pathology in a clinical sample. Neurology. 20I8;90(4):e282-e290.

I4 Maia LF, Kaeser SA, Reichwald J, et al. Changes in amyloidbeta and Tau in the cerebrospinal fluid of transgenic mice overexpressing amyloid precursor protein. Sci Transl Med. 2013;5(I94):I94re2.

I5 Schelle J, Hasler LM, Gopfert JC, et al. Prevention of tau increase in cerebrospinal fluid of APP transgenic mice suggests downstream effect of BACEI inhibition. Alzheimers Dement. 20I7;I3 (6):70I-709.

I6 De Felice FG, Wu D, Lambert MP, et al. Alzheimer's disease-type neuronal tau hyperphosphorylation induced by A beta oligomers. Neurobiol Aging. 2008;29(9):I334-1347.

I7 Sackmann C, Hallbeck M. Oligomeric amyloid-beta induces early and widespread changes to the proteome in human iPSC-derived neurons. Sci Rep. 2020;IO(I):6538.

I8 Barthelemy NR, Li Y, Joseph-Mathurin N, et al. A soluble phosphorylated tau signature links tau, amyloid and the evolution of stages of dominantly inherited Alzheimer's disease. Nat Med. 2020;26(3):398-407.

I9 Sato C, Barthelemy NR, Mawuenyega KG, et al. Tau kinetics in neurons and the human central nervous system. Neuron. 2018;97 (6):I284-I298. e7.

20 Bateman RJ, Xiong C, Benzinger TL, et al. Clinical and biomarker changes in dominantly inherited Alzheimer's disease. $N$ Engl $J$ Med. 2012;367(9):795-804.

2I Toledo JB, Xie SX, Trojanowski JQ, Shaw LM. Longitudinal change in CSF Tau and Abeta biomarkers for up to 48 months in ADNI. Acta Neuropathol. 2013;126(5):659-670.

22 Brunello CA, Merezhko M, Uronen RL, Huttunen HJ. Mechanisms of secretion and spreading of pathological tau protein. Cell Mol Life Sci. 2020;77(9):I72I-I744.

23 Ishiguro K, Ohno H, Arai H, et al. Phosphorylated tau in human cerebrospinal fluid is a diagnostic marker for Alzheimer's disease. Neurosci Lett. I999;270(2):9I-94. 
24 Janelidze S, Stomrud E, Smith R, et al. Cerebrospinal fluid ptau2I'7 performs better than p-taui8I as a biomarker of Alzheimer's disease. Nat Commun. 2020;II(I):I683.

25 Russell CL, Mitra V, Hansson K, et al. Comprehensive quantitative profiling of tau and phosphorylated tau peptides in cerebrospinal fluid by mass spectrometry provides new biomarker candidates. $J$ Alzheimers Dis. 2017;55(I):303-3I3.

26 Buerger K, Zinkowski R, Teipel SJ, et al. Differential diagnosis of Alzheimer disease with cerebrospinal fluid levels of tau protein phosphorylated at threonine 231. Arch Neurol. 2002;59(8):I267I272.

27 Barthelemy NR, Bateman RJ, Hirtz C, et al. Cerebrospinal fluid phospho-tau T2I7 outperforms TI8I as a biomarker for the differential diagnosis of Alzheimer's disease and PET amyloid-positive patient identification. Alzheimers Res Ther. 2020;I2(I):26.

28 Karikari TK, Emersic A, Vrillon A, et al. Head-to-head comparison of clinical performance of CSF phospho-tau TI8I and T2I7 biomarkers for Alzheimer's disease diagnosis. Alzheimers Dement. 2020.

29 Mielke MM, Aakre JA, Algeciras-Schimnich A, et al. Comparison of CSF phosphorylated tau I8I and 2I7 for cognitive decline. Alzheimers Dement. 202I.

30 Leuzy A, Janelidze S, Mattsson-Carlgren N, et al. Comparing the clinical utility and diagnostic performance of CSF P-TauI8I, PTau2I7, and P-Tau23I Assays. Neurology. 202I;97(I7):eI68IeI694.

3I Suarez-Calvet M, Karikari TK, Ashton NJ, et al. Novel tau biomarkers phosphorylated at TI8I, T2I7 or T23I rise in the initial stages of the preclinical Alzheimer's continuum when only subtle changes in Abeta pathology are detected. EMBO Mol Med. 2020: eI292I.

32 Uhlmann RE, Rother C, Rasmussen J, et al. Acute targeting of preamyloid seeds in transgenic mice reduces Alzheimer-like pathology later in life. Nat Neurosci. 2020;23(I2):I580-I588.

33 Horowitz PM, Patterson KR, Guillozet-Bongaarts AL, et al. Early Nterminal changes and caspase- 6 cleavage of tau in Alzheimer's disease. J Neurosci. 2004;24(36):7895-7902.

34 De Vos A. A novel conformational, phospho-Threonine 23I specific assay for CSF protein tau. J Prev Alzheimers Dis. 2016

35 Cselenyi Z, Jonhagen ME, Forsberg A, et al. Clinical validation of I8F-AZD4694, an amyloid-beta-specific PET radioligand. $J \mathrm{Nucl}$ Med. 20I2;53(3):4I5-424.

36 Pascoal TA, Shin M, Kang MS, et al. In vivo quantification of neurofibrillary tangles with [(I8)F]MK-6240. Alzheimers Res Ther. 20I8; IO(I):74.

37 Therriault J, Benedet AL, Pascoal TA, et al. Association of Apolipoprotein E epsilons With Medial Temporal Tau Independent of Amyloid-beta. JAMA Neurol. 2020;77(4):470-479.

38 Pascoal TA, Therriault J, Benedet AL, et al. I8F-MK-6240 PET for early and late detection of neurofibrillary tangles. Brain. 2020;I43 (9):28I8-2830.

39 Pascoal TA, Mathotaarachchi S, Shin M, et al. Amyloid and tau signatures of brain metabolic decline in preclinical Alzheimer's disease. Eur J Nucl Med Mol Imaging. 20I8;45(6):IO2I-IO3O.

40 Therriault J, Benedet A, Pascoal TA, et al. Determining Amyloidbeta positivity using [(I8)F]AZD4694 PET imaging. J Nucl Med. 2020.

4I Klunk WE, Koeppe RA, Price JC, et al. The Centiloid Project: standardizing quantitative amyloid plaque estimation by PET. Alzheimers Dement. 20I5;II(I):I-I5. eI-4.

42 Rowe CC, Jones G, Dore V, et al. Standardized expression of I8F$\mathrm{NAV}_{4} 6_{94}$ and IIC-PiB beta-amyloid PET results with the centiloid scale. J Nucl Med. 20I6;57(8):I233-I237.

43 Braak H, Braak E. Neuropathological stageing of Alzheimer-related changes. Acta Neuropathol. I991;82(4):239-259.

44 Braak H, Alafuzoff I, Arzberger T, Kretzschmar H, Del Tredici K. Staging of Alzheimer disease-associated neurofibrillary pathology using paraffin sections and immunocytochemistry. Acta Neuropathol. 2006;II2(4):389-404.

45 Mathotaarachchi S, Wang S, Shin M, et al. VoxelStats: a MATLAB package for multi-modal voxel-wise brain image analysis. Front Neuroinform. 2016;10:20.
46 Worsley KJ, Taylor JE, Tomaiuolo F, Lerch J. Unified univariate and multivariate random field theory. Neuroimage. 2004;23(Suppl I):Si89-Si95.

47 Wu JW, Herman M, Liu L, et al. Small misfolded Tau species are internalized via bulk endocytosis and anterogradely and retrogradely transported in neurons. J Biol Chem. 2013;288(3):I856I870.

48 Mirbaha H, Holmes BB, Sanders DW, Bieschke J, Diamond MI. Tau trimers are the minimal propagation unit spontaneously internalized to seed intracellular aggregation. J Biol Chem. 2015;290 (24):I4893-I4903.

49 Santos JRF, Bauer C, Schuchhardt J, et al. Validation of a prototype tau Thr23I phosphorylation CSF ELISA as a potential biomarker for Alzheimer's disease. J Neural Transm. 20I9;126(3):339-348. (Vienna).

50 Spiegel J, Pirraglia E, Osorio RS, et al. Greater specificity for cerebrospinal fluid P-tau23I over P-taur8I in the differentiation of healthy controls from Alzheimer's disease. J Alzheimers Dis. 2016;49(I):93-I00.

5I Blennow K, Vanmechelen E, Hampel H. CSF total tau, Abeta42 and phosphorylated tau protein as biomarkers for Alzheimer's disease. Mol Neurobiol. 200I;24(I-3):87-97.

52 Glodzik-Sobanska L, Pirraglia E, Brys M, et al. The effects of normal aging and ApoE genotype on the levels of CSF biomarkers for Alzheimer's disease. Neurobiol Aging. 2009;30(5):672-68I.

53 Kidemet-Piskac S, Babic Leko M, Blazekovic A, et al. Evaluation of cerebrospinal fluid phosphorylated tau23I as a biomarker in the differential diagnosis of Alzheimer's disease and vascular dementia. CNS Neurosci Ther. 2018;24(8):734-740.

54 Mitchell AJ. CSF phosphorylated tau in the diagnosis and prognosis of mild cognitive impairment and Alzheimer's disease: a metaanalysis of 5I studies. J Neurol Neurosurg Psychiatry. 2009;80 (9):966-975.

55 Augustinack JC, Schneider A, Mandelkow EM, Hyman BT. Specific tau phosphorylation sites correlate with severity of neuronal cytopathology in Alzheimer's disease. Acta Neuropathol. 2002;I03 (I):26-35.

56 Ercan-Herbst E, Ehrig J, Schondorf DC, et al. A post-translational modification signature defines changes in soluble tau correlating with oligomerization in early stage Alzheimer's disease brain. Acta Neuropathol Commun. 20I9;7(I):I92.

57 Castellani RJ, Perry G. Tau biology, tauopathy, traumatic brain injury, and diagnostic challenges. J Alzheimers Dis. 2019;67 (2):447-467

58 Amniai L, Barbier P, Sillen A, et al. Alzheimer disease specific phosphoepitopes of Tau interfere with assembly of tubulin but not binding to microtubules. FASEB J. 2009;23(4):II46-II52.

59 Palmqvist S, Scholl M, Strandberg O, et al. Earliest accumulation of beta-amyloid occurs within the default-mode network and concurrently affects brain connectivity. Nat Commun. 20I7;8(I):I2I4 .

6o Ashton NJ, Pascoal TA, Karikari TK, et al. Plasma p-tau23I: a new biomarker for incipient Alzheimer's disease pathology. Acta Neuropathol. 202I;I4I(5):709-724.

6I Malarte ML, Nordberg A, Lemoine L. Characterization of MK6240, a tau PET tracer, in autopsy brain tissue from Alzheimer's disease cases. Eur J Nucl Med Mol Imaging. 2020.

62 Blennow K, Hampel H. CSF markers for incipient Alzheimer's disease. Lancet Neurol. 2003;2(I0):605-6I3.

63 Mattsson-Carlgren N, Andersson E, Janelidze S, et al. Abeta deposition is associated with increases in soluble and phosphorylated tau that precede a positive Tau PET in Alzheimer's disease. Sci Adv. 2020;6(I6):eaaz2387.

64 Gordon BA, Blazey TM, Christensen J, et al. Tau PET in autosomal dominant Alzheimer's disease: relationship with cognition, dementia and other biomarkers. Brain. 20I9;I42(4):I063-I076.

65 Pooler AM, Phillips EC, Lau DH, Noble W, Hanger DP. Physiological release of endogenous tau is stimulated by neuronal activity. EMBO Rep. 20I3;I4(4):389-394.

66 Sutphen CL, McCue L, Herries EM, et al. Longitudinal decreases in multiple cerebrospinal fluid biomarkers of neuronal injury in symptomatic late onset Alzheimer's disease. Alzheimers Dement. 2018;I4(7):869-879. 